\title{
Cognitive Trait Model and Divergent Associative Learning
}

\author{
Taiyu Lin, Kinshuk ${ }^{1}$ and Sabine Graf ${ }^{2}$ \\ Massey University, New Zealand, taiyu.lin@gmail.com \\ ${ }^{1}$ Athabasca University, Canada, kinshuk@ieee.org \\ ${ }^{2}$ Vienna University of Technology, Austria, sabine.graf@ieee.org
}

\begin{abstract}
Cognitive trait model (CTM) is a student model that aims to create profiles of learners' cognitive traits. Divergent associative learning (DAL) denotes the characteristic of learning that develops links between new and existing concepts. Relationships of DAL to divergent thinking and associative learning are examined in this paper. Manifestations of DAL are extracted from literature and used in CTM to create approximations of learners' DAL. A web-based tool called Web-DAL was developed to measure DAL psychometrically in an empirical study. In this study, the comparison of the approximations of DAL and the psychometric data shows a promising sign of using the proposed CTM to profile learners' DAL.
\end{abstract}

\section{Introduction}

Cognitive trait model (CTM) is a student model that aims to create profiles of learners' cognitive traits by analysing learners' interactions with learning systems [1]. Two cognitive traits, working memory and inductive reasoning have been studied previously [1]. In this paper, we investigate another cognitive trait called divergent associative learning.

\section{Cognitive Trait Model}

Cognitive trait model (CTM), a student model that profiles students according to their cognitive traits, is structured as shown in Figure 1 [1]. The learner interface presents the learning system to the learners and enables interactions. Series of learner actions are then passed on to the Action History and stored. Performance-based student models represent learners' domain competence and model the problem-solving process that the learners undertake.

Certain learner behaviours, called manifestations of trait (MOTs) in this study, can be used to infer cognitive traits of learners. Each MOT is a piece of an interaction pattern that manifests certain learner cognitive trait (e.g. low DAL). The MOT Detector Component detects those MOTs among the series of actions recorded in the Action History Component.

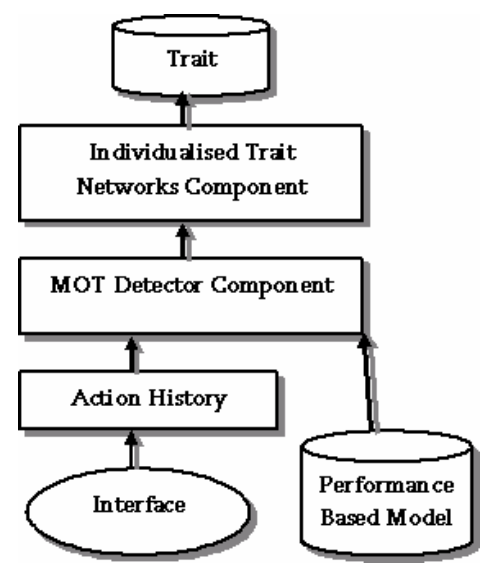

Figure 1: Cognitive Trait Model

The Individualised Trait Network (ITN) Component assigns each detected MOT to one of the ITNs. Each ITN represents one cognitive trait and has a number of weighted nodes in itself. The number of nodes depends on the number of MOTs of the cognitive trait the ITN is representing. The weights of nodes get updated after each learning session, similar to how a neural network updates itself and learns [3]. The results of executions of each ITN are then saved in the Trait Model via the Trait Model Gateway.

\section{Divergent Associative Learning}

Divergent associative learning (DAL) denotes the characteristic of learning that develops links between new and existing concepts. It inherits certain characteristics from both divergent thinking and associative learning. This section is devoted to study the similarities and differences of DAL to divergent thinking and associative learning. 
Divergent thinking often manifests itself by creating many responses to a single stimulus and is often related to creativity [4]. It is opposite to convergent thinking, which manifests itself by arriving at the single correct solution from many potential options.

Many of the studies about associative learning are based on the assumption that association can be formed between simple events or stimuli [5]. Associations between the simple events, such as lights, tones and food, are the targets to be learned. One end of the association is often called the stimulus and the other the response [8]. The stimulus and the response constitute a pair to be learned and task of this type is often called paired-associate learning [8].

Most of the researches on divergent thinking focused on production - that is to create/produce some creative artefacts no matter physical or mental. In this study, it is more important to know how divergent thinking can help in the learning context. In the view of associative learning, a new concept associated with existing concepts during the learning process gains higher potential for future recall. It is because if any of the associated existing concepts is activated, the new concept can get activated too [9]. Therefore, instead of focusing on the production of new ideas, DAL creates divergent-style associations from the new concept to existing concepts.

DAL is different from divergent thinking in two ways: firstly, the end products of divergent thinking are new concepts/ideas whereas the end products of the DAL are new associations between the new concept and existing concepts. Secondly, the focus of divergent thinking is directed forwards into an unknown space in which novel and original ideas could be (and are expected to be) discovered. On the other hand, the focus of DAL is directed backwards to what has been learned before, i.e., searching for suitable candidate concepts to associate to.

In addition of creating associations between the given stimuli and responses in traditional associative learning [8], DAL requires also effort to search for suitable targets. Traditional associative learning, especially the pair-associate paradigm, only requires the learning of the one-to-one associations between given stimuli and responses, whereas DAL focuses on learning of one-to-many associations.

\section{Web-DAL}

A test has been devised in this research to measure learners' DAL and it is called Web-DAL. Web-DAL is a computerised test, and the prefix "Web" indicates its ability to be deployed on the Internet.
A subject taking the Web-DAL is first presented with a new concept. After learning the new concept, the subject is asked to write down as many related concepts as possible in one minute. The selection of new concept is from a domain that the target subjects are familiar with. Selection of such a domain removes the possible bias that some domains could be remote for some subjects.

Experiment in [4] allowed the subjects for three minutes to write down as many uses of three objects as possible. In the Web-DAL task, however, there is only one new concept. After preliminary evaluation by us, one minute time frame was deemed enough to differentiate subjects with high and low DAL.

The difference of Web-DAL and other divergent thinking tests, such as Alternate Uses Test (AUT) [19], is that there is learning involved in Web-DAL but not in AUT. In AUT, subjects simply were told to create as many keywords as they could think of relevant to a daily object. On the other hand, in Web-DAL, subjects are required to learn the new concept and then create divergent associations to existing knowledge that are pertinent to the new concept.

The difference of Web-DAL to other associative learning tests, such as paired-associate task [8], is the duration of time between learning and testing. In paired-associate task, subjects are tested shortly (minutes or hours) after they learn the associations. However, the duration between learning and testing is much longer (weeks or months) in Web-DAL. Except for students cramming for exams, we believe that the application of the long term learning is more common than that of the short term learning. Tasks, such as in Web-DAL, that test the long term associative learning can better reflect a subject's usual performance than tasks, such as paired-associate, that test on short term associative learning.

\section{Empirical Study}

In the empirical study carried out in this research, cognitive trait model used the six MOTs of DAL described in section 3 to create approximations of students' DAL. The approximation was compared to the performances in the Web-DAL task to evaluate the proposed approach. Details of the empirical study are described next.

There were a total of 14 subjects participated in this empirical study. The participants were students of Massey University in New Zealand studying in Information System course. Subjects in this study took the Web-DAL task and used a web-based learning system that taught Information Technology 
Infrastructure and PHP programming. The learning system used cognitive trait model to create approximations of DAL of learners.

Subjects in this study were each given a set of userID and password to login to the learning system and Web-DAL. In the learning system, a subject could just login and start using it. They could read the descriptions of the concepts in the domain or take quizzes. Quizzes were multiple-choice questions.

For the Web-DAL task, subjects followed the procedure described in section 4 .

The index of performance in the Web-DAL task is the total number of answer items and is represented by the variable fluency. Another variable sumOrg is also calculated by the sum of each answer item's originality score which is the inverse of its frequency of occurrence in the entire sample. The approximations of DAL in CTM are represented by the variable DALApp.

$D A L A p p$ is found to be significantly correlated to both sumOrg (rho=0.653, Sig. $=0.06$ ) and fluency (rho=0.499, Sig. $=0.035$ ). These correlations support the use of cognitive trait model to create DAL profile of learners.

\section{Summary}

Cognitive trait model is a student model that creates cognitive profile of learners. The cognitive profile in this paper is about divergent associative learning (DAL). DAL denotes the characteristic of learning that develops links between new and existing concepts. Six manifestations of trait (MOTs) of DAL are used in this study: associative hierarchy, classification ability, versatile navigation, relevance filtering, domain performance and working memory.

A learning system was created where students' behaviours in the learning system were logged into the database. The six MOTs were then used in CTM to approximate the DAL of learners from learner behaviours. Approximations of DAL were then used to compare with results obtained from the psychometric Web-DAL task. Statistical analysis showed significant correlations between the two scores and supports the use of CTM to create DAL profiles of learners.

\section{References}

[1] Kinshuk, T. Lin, A. Patel, "User Exploration Based Adaptation in Adaptive Learning Systems". International Journal of Information Systems in Education, 1 (1), 2003, 22-31 (ISSN 1348-236X).

[3] Y. Bar-Yam, Dynamics of complex systems. Perseus Books, HarperCollins, Canada, 1999.

[4] O. Vartanian, C. Martindale, and J. Kwiatkowski, "Creativity and inductive reasoning: The relationship between divergent thinking and performance on Wason's 24-6 task", The quarterly journal of experimental psychology, 56a, 2003, 641-656.

[5] C. Bonardi, "Conditional learning: An associative analysis”. In Nestor A Schmajuk, and Peter C Holland (eds), Occasion setting: Associative learning and cognition in animals. American Psychological Association, Washington, DC, USA, 1998.

[8] L. M. Horowitz, \& A. M. Gordon, "Associative symmetry and second language learning”, Journal of Educational Psychology, 63 (3), 1972, 287-294.

[9] J. R. Anderson, "A spreading activation theory of memory”, Journal of Verbal Learning \& Verbal Behavior, 22 (3), Jun 1983, 261-295.

[10] S. A. Mednick, The associative basis of the creative process. Psychological Review, 69, 1962, 220-232.

[11] R. J. Sternberg, \& M. K. Gardner, "Unities in inductive reasoning”, Journal of Experimental Psychology: General, 112 (1), 1983, 80-116.

[13] H. Huai, Cognitive style and memory capacity: effects of concept mapping as a learning method. Doctoral Thesis, Oct 2000, Twente University, The Netherlands.

[14] H. J. Eysenck, "Creativity and personality: Suggestions for a theory”, Psychological Inquiry, 4 (3), 1993, 147-178.

[15] N. Ryder, L. Pring, \& B. Hermelin, "Lack of Coherence and Divergent Thinking: Two Sides of the Same Coin in Artistic Talent?” Current Psychology, 21 (2), 2002, 168-176. [16] J. F. Feldhusen, "Creativity: the knowledge base and children”, High Ability Studies, 13 (2), 2002, 179-183.

[18] M. Bahar, and M. H. Hansell, "The relationship between some psychological factors and their effect on the performance of grid questions and word association tests”, Educational Psychology, 20 (3), 2000, 349-364.

[19] M. A. Wallach, \& N. Kogan, Modes of thinking in young children: A study of the creativity-intelligence distinction. Holt, Rinehart, \& Winston, New York, 1965. 